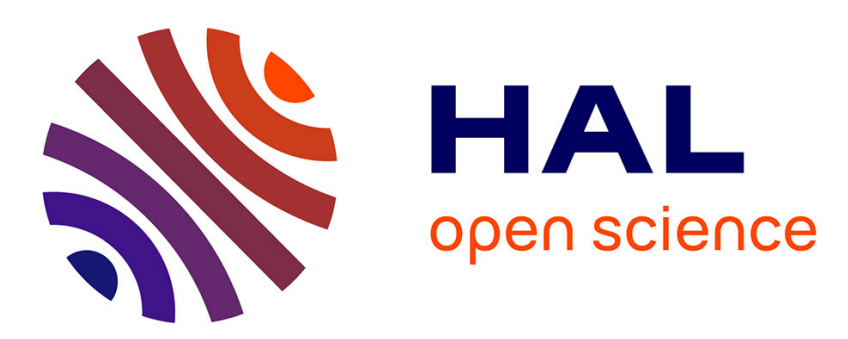

\title{
Fault-tolerant control design with respect to actuator health degradation: An LMI approach
}

Ahmed Khelassi, Didier Theilliol, Philippe Weber, Jean-Christophe Ponsart

\section{To cite this version:}

Ahmed Khelassi, Didier Theilliol, Philippe Weber, Jean-Christophe Ponsart. Fault-tolerant control design with respect to actuator health degradation: An LMI approach. IEEE Multi-Conference on Systems and Control, MSC 2011, Sep 2011, Denver, Colorado, United States. pp.CDROM. hal00653108

\section{HAL Id: hal-00653108 \\ https://hal.science/hal-00653108}

Submitted on 17 Dec 2011

HAL is a multi-disciplinary open access archive for the deposit and dissemination of scientific research documents, whether they are published or not. The documents may come from teaching and research institutions in France or abroad, or from public or private research centers.
L'archive ouverte pluridisciplinaire HAL, est destinée au dépôt et à la diffusion de documents scientifiques de niveau recherche, publiés ou non, émanant des établissements d'enseignement et de recherche français ou étrangers, des laboratoires publics ou privés. 


\title{
Fault-tolerant control design with respect to actuator health degradation: An LMI approach
}

\author{
Ahmed Khelassi, Didier Theilliol, Philippe Weber and Jean-Christophe Ponsart
}

\begin{abstract}
The active fault-tolerant control approach relies heavily on the occurred faults. In order to improve the safety of the reconfigurable system, a methodology to incorporate actuator health in the fault-tolerant control design is proposed for a tracking problem. Indeed, information about actuator health degradation due to the applied control is considered in addition to fault estimation. The main objective is to design a fault-tolerant control system which guarantees a high overall system reliability and dependability both in nominal operation and in the presence of faults. Such an objective is achieved by a control performance index, which is proposed based on system reliability analysis. The fault-tolerant controller is synthesized by using a linear matrix inequality approach.
\end{abstract}

\section{INTRODUCTION}

The safety-critical nature of embedded systems used in air-craft, space, tactical, and automotive applications, mandates that the functionality for which they are designed be performed even in the presence of component faults. Thus, safety-critical systems should be able to adapt and compensate the component faults in systematic way. These types of adaptable systems are known as fault-tolerant control systems (FTCS). The aim of FTCS is to keep plant available by the ability to achieve the objectives assigned in the faulty situations and to accept reduced performance when critical faults occur [1].

Various approaches for FTCS design have been proposed in the literature. Overviews on the development of FTCS have been provided in survey papers and books as for example in [14] and [8]. The FTCS design approaches can be classified under two types, the passive approaches and the active approaches [14]. In the passive approaches, the same controller is used for the normal case as well as for the faulty cases where, a presumed set of process component faults are considered in the design stage. However, active FTC requires an on-line Fault Detection and Diagnosis (FDD) process and control reconfiguration mechanism [14].

Therefore, it is important to enhance the system safety not only by improving reliability of individual components, or by designing control systems to compensate the effect of faults but also, by taking into consideration the degradation of components health in the controller design [6]. Indeed, as illustrated in [1], fault-tolerant control can improve the overall system reliability, because with an effective control input

The authors are with the Centre de Recherche en Automatique de Nancy, CRAN-CNRS UMR 7039, Nancy University, BP 70239, 54500 Vandeuvre Cedex, France. (E-mail: ahmed.khelassi@cran.uhp-nancy.fr, didier.theilliol@cran.uhp-nancy.fr, philippe.weber@cran.uhp-nancy.fr). This work was supported by the SAFFE project 2010-2011 (Ageing Management in fault-tolerant control system design project) from GIS 3SGS - France (https://www.gis-3sgs.fr/). management, the overall system remains operational for a longer duration with an acceptable performance requirement. This problem is more considerable in practical safety-critical systems and in flight control systems where a catastrophic consequences from degraded functional operation can occur [14].

Some research works have introduced reliability analysis for FTCS. [11] and [12] have used Markov models to dictate the system reliability where subsystems are supposed to reach two states: intact (available) of failed (unavailable) states. [9] and [10] the authors proposed a sensor and actuator reconfiguration strategy based on physical redundancy. The reliability analysis provides some informations to select the optimal reconfigurable set. In a similar way, [5] has considered the reliability of sensor faults in the filtering design issue. A reconfiguration mechanism of FTC strategy incorporating reliability analysis under a dynamic behavior constraints has been proposed in [4] .

In this paper, informations about actuator health are integrated in the fault-tolerant control design. The proposed design contribute to a fault-tolerant controller which achieves the control objective with a high overall system reliability. Indeed, such design improves the dependability of the system and keeps the set of the actuators available as long as possible by minimizing the use of the more sensitive actuators. The degradation of actuator health is evaluated by estimating the impact of the required load on the actuator life time. In this context, the solution of fault-tolerant tracking problem incorporating actuator health is proposed by using an LMI approach.

This paper is organized as follows. In section II, the faulty system model and the fault-tolerant tracking control problem are presented. In section III, a performance index for control design is proposed with respect to actuator health level. Section IV, a solution of fault-tolerant control tracking problem with respect to overall system reliability is proposed. In section $\mathrm{V}$, a numerical example of an $\mathrm{F}$ 16 aircraft model and its simulation results are given and compared to a classical control performance index. Some concluding remarks are given in section VI.

\section{PRELIMINARIES AND PROBLEM FORMULATION}

Let us consider the linear time-invariant model given by:

$$
\begin{aligned}
& \dot{x}(t)=A x(t)+B u(t) \\
& y(t)=C x(t) \\
& z(t)=S y(t)
\end{aligned}
$$


where $A \in \mathbb{R}^{n \times n}, B \in \mathbb{R}^{n \times m}$ and $C \in \mathbb{R}^{p \times n}$ are respectively, the state, the control and the output matrices. $x \in \mathbb{R}^{n}$ is the system state, $u \in \mathbb{R}^{m}$ is the control input, $y \in \mathbb{R}^{p}$ is the system output. $S \in R^{l \times p}$ is a known constant matrix used to select the output required to track the reference signal. $z(t) \in \mathbb{R}^{l}$ is the controlled output and $(A, B)$ is stabilizable.

In this study, the considered faults are loss of control effectiveness where the system (1) can be written in degraded operating mode as follows:

$$
\begin{aligned}
& \dot{x}(t)=A x(t)+B_{f} u(t) \\
& y(t)=C x(t) \\
& z(t)=S y(t)
\end{aligned}
$$

The matrix $B_{f}$ is written according to the nominal control input matrix $B$ and the control effectiveness factors $\omega_{i} \in$ $\left[\begin{array}{ll}0 & 1\end{array}\right], i=1, \ldots, m$, as follows:

$$
B_{f}=B \omega, \omega=\operatorname{diag}\left(\left[\omega_{1}, \omega_{2}, \ldots, \omega_{m}\right]\right)
$$

Indeed, if $\omega_{i}=1$, then the $i^{t h}$ actuator is considered to be fault-free. Nevertheless, when $0<\omega_{i} \leq 1$, the considered fault is a partial loss of control effectiveness. Moreover, when $\omega_{i}=0$ failure is considered and the actuator is out of order.

For the faulty system described by (2), the fault-tolerant control design problem is to determine a controller such that:

i) During normal operating mode, the closed-loop system is stable and the output $z(t)$ tracks the reference signal $y_{r}(t)$ without steady-state error:

$$
\lim _{t \longrightarrow \infty} e(t)=0, \quad e(t)=y_{r}(t)-S y(t)
$$

In fact, this study deals with active fault-tolerant control AFTC.

ii) After faults diagnosis, the closed-loop system is still stable and the required output $z(t)$ tracks the reference signal $y_{r}(t)$ with an acceptable lower level of tracking performance.

Is is known that integrator control can be added in the closed-loop system to eliminate the steady state tracking error. The integral term can be constantly calculating the value of the control required at the set-point to force the error to go to zero. To accomplish the design of the control system for the integral and the original state vector, an augmented model is considered by combining (2) and (4) as follows:

$$
\left[\begin{array}{c}
\dot{\varepsilon}(t) \\
\dot{x}(t)
\end{array}\right]=\left[\begin{array}{cc}
0 & -S C \\
0 & A
\end{array}\right]\left[\begin{array}{l}
\varepsilon(t) \\
x(t)
\end{array}\right]+\left[\begin{array}{l}
0 \\
B
\end{array}\right] \omega u(t)+\left[\begin{array}{l}
I \\
0
\end{array}\right] y_{r}(t)
$$

where $\varepsilon(t)=\int_{0}^{t} e(\tau) d \tau$ is the integral error state.

By considering $\bar{x}(t)=\left[\begin{array}{ll}\varepsilon^{T}(t) & x^{T}(t)\end{array}\right]^{T}$, the augmented system (5) can be described as follows:

$$
\dot{\bar{x}}(t)=\bar{A} \bar{x}(t)+\bar{B} \omega u(t)+G y_{r}(t)
$$

where $\bar{A}=\left[\begin{array}{cc}0 & -S C \\ 0 & A\end{array}\right], \quad \bar{B}=\left[\begin{array}{cc}0 & B\end{array}\right]^{T}, G=\left[\begin{array}{cc}I & 0\end{array}\right]^{T}$.
Indeed, the performance index which the designed controller should minimize is written as follows:

$\mathcal{J}_{t}=\int_{0}^{t}\left[\varepsilon(t)^{T} Q_{1} \varepsilon(t)+x(t)^{T} Q_{2} x(t)+u(t)^{T} \omega R \omega u(t)\right] d t$

where $Q_{1} \in \mathbb{R}^{l \times l}, Q_{2} \in \mathbb{R}^{n \times n}$ are semidefinite matrices and $R \in \mathbb{R}^{m \times m}$ is a positive definite matrix.

Several fault-tolerant control approaches have been proposed in the literature but without interest in the improvement of the reconfigurable system life time. The main contribution of this work is to consider the overall system reliability as a principle objective which guides the design of the fault-tolerant controller. Thus, information about actuator health can be introduced in the performance index (7) in order to guarantee that the control efforts are distributed to the system by taking into account the reliability of the actuators. The aim is design a fault-tolerant controller that maximizes the overall system reliability. The stability and tracking problem will be solved, both in the nominal case and in the faulty conditions, by using an LMI approach.

\section{CONTROL PERFORMANCE INDEX BASED ON RELIABILITY ANALYSIS}

One motivation to integrate information about actuator health in the controller design is to improve the life time of the reconfigurable systems which is quantified by the overall system reliability estimation. Let us consider the following definitions:

Definition 1: Actuator health is a measure that can be affected by several parameters and factors. In this study, actuator reliability and loss of control effectiveness are both considered.

Definition 2: Reliability $R(t)$ is defined as the probability that units, components, equipments and systems will accomplish their intended function for a specified period of time under some operating conditions and specific environments, [3].

In the useful period of life, the component can be characterized at a given time $t$ by a baseline reliability measure $R^{0}(t)$. In following, $R_{i}^{0}(t)$ will be assigned to the reliability of the $i^{\text {th }}$ actuator obtained under nominal operating conditions in the useful period of life such as:

$$
R_{i}^{0}(t)=\exp \left(-\lambda_{i}^{0} t\right), \quad i=1 \ldots m
$$

where in the reliability engineering field, $\lambda_{i}^{0}$ is a baseline failure rate of the $i^{t h}$ component defined under a nominal load level called stress level.

However, a realistic health measurement should also include the trend of actuator degradation according to the variation of the operating conditions. Indeed, in many situations and especially in the considered study, failure rates are obtained from actuators under different levels of load depending on the applied control input. Several mathematical models have been developed to introduce the impact of the variable load level in the reliability estimation [3]. Proportional hazard model firstly proposed in [2] is adopted in this paper. 
Definition 3: For a variable functional condition, the actuator reliability can be estimated according to the baseline failure rate as follows:

$$
\begin{aligned}
& R_{i}(t)=\exp \left(-\lambda_{i} t\right) \\
& \lambda_{i}=\lambda_{i}^{0} \times g_{i}(\ell, \vartheta)
\end{aligned}
$$

where $\lambda_{i}^{0}$ represents the baseline failure rate (nominal failure rate) for the $i^{\text {th }}$ actuator and $g_{i}(\ell, \vartheta)$ is a load function (independent of time) which represents the effects of stress on the component failure rate $\ell$ represents an image of the applied load and $\vartheta$ is a component parameter.

Assumption 1: In this study, the exponential distribution for reliability estimation is considered. The load function (9) will be defined according to the load applied on the actuator until the end of the mission $t=t_{M}$. The component reliability measure can be evaluated for $t=t_{M}$ and noted $R\left(t_{M}\right)$.

Different definitions of the load function $g_{i}(\ell, \vartheta)$ exist in the literature. However, the exponential form is commonly used [4]. In this study, the load function is defined according to the root-mean-square of the applied control input as follows:

$$
g_{i}(\ell, \vartheta)=\exp \left(\beta_{i} \int_{0}^{t_{M}} u_{i}^{2}(t) d t\right), \quad i=1, \ldots, m
$$

where $\beta_{i}$ is an actuator parameter defined as follows:

$$
\beta_{i}=\left(t_{M}\left(\bar{u}_{i}-\underline{u}_{i}\right)\right)^{-1}, \quad i=1, \ldots, m
$$

where $\bar{u}_{i}=$ and $\underline{u}_{i}$ are the upper and lower saturation bound of $u_{i}$ respectively.

Property 1: Based on (9) and (10), the actuator health at a given time $t_{M}$ is modeled as a function of the applied load where the actuator reliability degradation increases for a large control input.

Indeed, the actuator reliability $R_{i}\left(t_{M}\right)$ can be estimated depending on the baseline reliability level $R_{i}^{0}\left(t_{M}\right)$ as follows:

$$
\begin{aligned}
& R_{i}\left(t_{M}\right)=\alpha_{i}\left(u_{i}\right) R_{i}^{0}\left(t_{M}\right), \quad i=1, \ldots m \\
& \alpha_{i}\left(u_{i}\right)=\exp \left(-\lambda_{i}^{0} \beta_{i}\left\|u_{i}\right\|^{2}\right)
\end{aligned}
$$

where, $\|$.$\| is the Euclidien norm. \alpha_{i}\left(u_{i}\right) \leq 1$ represents the rate of reliability degradation due to the applied load during the mission. In fact, the following relation is satisfied: if $\left\|u_{i}\left(t_{M}\right)\right\|^{2} \rightarrow 0: R\left(t_{M}\right) \rightarrow R^{0}\left(t_{M}\right)$.

Lemma 1: For a system composed by $m$ redundant actuators, the overall system reliability $R g\left(t_{M}\right) \longrightarrow R g^{\max }$ for an effective control inputs $u^{*}(t)=-K x(t) \in \mathcal{U}$ that stress/solicit less the more reliable actuators in the control efforts distribution and satisfy the following condition as close as possible:

$$
\mathcal{U}=\left\{u^{*}(t) \mid \min \frac{\alpha_{i}\left(u_{i}\right)}{\alpha_{j}\left(u_{j}\right)} \text { if } \frac{\lambda_{i}^{0}}{\lambda_{j}^{0}}<1\right\}
$$

where $K$ is the nominal controller, $i \in[1, \ldots, m], j \in$ $[1, \ldots, m]$ and $i \neq j . R g^{\max }$ is the optimal value of overall system reliability that can be obtained at $t=t_{M}$.

Proof: Systems composed by $m$ redundant actuators can be presented by a parallel scheme of Reliability Block
Diagram (RBD). The overall system reliability under nominal conditions $R g^{0}\left(t_{M}\right)$ can be obtained as follows:

$$
R g^{0}\left(t_{M}\right)=1-\prod_{k=1}^{m}\left(1-R_{k}^{0}\left(t_{M}\right)\right)
$$

The sensitivity of the overall system reliability versus the $i^{\text {th }}$ actuator $s_{i}$ can be evaluated as:

$$
\begin{aligned}
s_{i} & =\frac{d R g^{0}\left(t_{M}\right)}{d R_{i}^{0}\left(t_{M}\right)}=\prod_{k=1 ; k \neq i}^{m}\left(1-R_{k}^{0}\left(t_{M}\right)\right) \\
& =\prod_{k=1}^{m}\left(1-R_{k}^{0}\left(t_{M}\right)\right)\left(1-R_{i}^{0}\left(t_{M}\right)\right)^{-1}
\end{aligned}
$$

It can be shown that, if $R_{i}^{0}>R_{j}^{0}$ (or $\lambda_{i}^{0}<\lambda_{j}^{0}$ ) then $s_{i}<s_{j}$. This result means that a small degradation $\alpha_{i}\left(u_{i}\right)$ of the more sensitive actuator $i$ causes a large degradation of the overall system reliability $R g\left(t_{M}\right)$. So, the optimal value of the overall system reliability under a variable functional condition can be obtained for control inputs as follows:

$$
u^{*}=\arg \min \frac{\alpha_{i}\left(u_{i}\right)}{\alpha_{j}\left(u_{j}\right)}: R g\left(t_{M}\right) \longrightarrow R g^{\max } \text { s.t. } \frac{\lambda_{i}^{0}}{\lambda_{j}^{0}}<1
$$

which limit the use of the less reliable actuators in the control efforts distribution.

The actuators failure rates can be ordered from the less sensitive actuator to the more sensitive as follows:

$$
l^{0}=\left\{\lambda_{m \underline{s}}^{0}, \lambda_{m \underline{s}+1}^{0}, \lambda_{m \underline{s}+2}^{0}, \ldots, \lambda_{p \bar{s}}^{0}\right\}
$$

where $p \bar{s}$ and $m \underline{s}$ are respectively the indexes of the most sensitive and the less sensitive actuator with $p \bar{s}:=m \underline{s}+m-$ 1 . The associated degradation rates $\alpha_{i}\left(u_{i}\right)$ can be re-arranged also as follows:

$$
\Theta=\left\{\alpha_{m \underline{s}}(u), \alpha_{m \underline{s}+1}(u), \alpha_{m \underline{s}+2}(u) \ldots, \alpha_{p \bar{s}}(u)\right\}
$$

where to simplify the notation, $\alpha_{i}\left(u_{i}\right):=\alpha_{i}(u)$ is adopted.

In order to design an effective fault-tolerant controller which guarantees a high overall system reliability taking into consideration the actuator health, the control inputs delivered by the controller should minimize the degradation rate of the most sensitive actuators and maximize the less sensitive for each subset $\left(\alpha_{m s+k-1}(u), \alpha_{m s+k}(u)\right)$ as close as possible. This objective can be reformulated as follows:

$$
\arg \min _{u} \sum_{k=1}^{m-1}\left(\frac{\alpha_{m \underline{s}+k}(u)}{\alpha_{m \underline{s}+k-1}(u)}\right), \quad k=1, \ldots,(m-1)
$$

Based on (12) associated with Taylor approximation $\left(\alpha_{i}(u)<1\right)$, the optimization objective $(16)$ can be written as follows,

$$
\begin{aligned}
& \arg \min _{u} \sum_{k=1}^{m-1}\left(\left\|u_{m \underline{s}+k-1}\right\|^{2}-\frac{\lambda_{m \underline{s}+k}^{0} \beta_{m \underline{s}+k}}{\lambda_{m \underline{s}+k-1}^{0} \beta_{m \underline{s}+k-1}}\left\|u_{m \underline{s}+k}\right\|^{2}\right) \\
&= \arg \min _{u}\left(\left\|u_{m \underline{s}}\right\|^{2}-\rho_{m \underline{s}+1}\left\|u_{m \underline{s}+1}\right\|^{2}\right)+ \\
&\left(\left\|u_{m \underline{s}+1}\right\|^{2}-\rho_{m \underline{s}}+2\left\|u_{m \underline{s}+2}\right\|^{2}\right)+\ldots \\
& \quad \ldots+\left(\left\|u_{m \underline{s}+m-2}\right\|^{2}-\rho_{p \bar{s}}\left\|u_{p \bar{s}}\right\|^{2}\right)
\end{aligned}
$$

which is equivalent to,

$$
\arg \min _{u} \sum_{i=1}^{m} r_{i}\left\|u_{i}\right\|^{2}=\arg \min _{u} \sum_{i=1}^{m} r_{i} \int_{0}^{t} u_{i}^{2}(t) d t
$$


where

$$
\begin{cases}r_{i}=1 & i=m \underline{s} \\ r_{i}=-\rho_{i} & i=p \bar{s} \\ r_{i}=1-\rho_{i} & p \bar{s}<i<m \underline{s}\end{cases}
$$

and

$$
\rho_{i}=\lambda_{i}^{0} \beta_{i}\left(\lambda_{i-1}^{0} \beta_{i-1}\right)^{-1}<1,
$$

The objective function (18) can be written as:

$$
\begin{gathered}
\arg \min _{u} \int_{0}^{t}\left(r_{1} u_{1}^{2}(t)+r_{2} u_{2}^{2}(t)+\ldots+r_{m} u_{m}^{2}(t)\right) d t \\
=\arg \min _{u} \int_{0}^{t} u(t)^{T} \mathcal{R} u(t) d t
\end{gathered}
$$

where, $\mathcal{R}=\operatorname{diag}\left(\left[r_{1}, r_{2}, \ldots, r_{m}\right]\right)$

However, in order to reformulate the function objective (21) such (19) and (20), as an index control performance with respect to the actuator health, the following performance index is proposed:

$$
\arg \min _{u} \int_{0}^{t} u(t)^{T}\left(\mathcal{R}+\sigma I_{m}\right) u(t) d t=\int_{0}^{t} u(t)^{T} \overline{\mathcal{R}} u(t) d t
$$

where $\sigma>1$ is introduced in the criterion to ensure the convexity of the optimization problem with $\overline{\mathcal{R}} \succ 0, \forall \sigma>1$. In fact, $\sigma$ is an adapted parameter used in the design stage to choose the desired dynamic of the closed-loop system defined by $A, B, C, Q$ and $\overline{\mathcal{R}}$ with respect to the actuators health levels.

To design an affective fault-tolerant controller which maximizes the overall system reliability, the classical performance index (7) is reformulated as in the following proposition:

Proposition 1: To design an effective fault-tolerant control system by taking into account the actuator health, the controller can be synthesized in order to minimize the upper bound of the following performance index:

$\mathcal{J}_{t}^{*}=\int_{0}^{t}\left[\varepsilon(t)^{T} Q_{1} \varepsilon(t)+x(t)^{T} Q_{2} x(t)+u(t)^{T} \omega \overline{\mathcal{R}} \omega u(t)\right] d t$

where $\overline{\mathcal{R}} \succ 0$ is a diagonal matrix designed based on actuators failure rates as in (19) and (22).

\section{ACtive FAULT-TOLERANT SCHEME FOR A RELIABLE DESIGN}

A solution to design an effective active fault-tolerant controller which minimizes the performance index (23) (both in the nominal case $\omega=I_{m}$ and after fault occurrence) is presented for the tracking problem. In fact, the active faulttolerant methods involve the introduction of an FDI module in the control loop. We assume that the fault is detected at $t=t_{f}$ and the reconfigurable control inputs can be applied at time $\tau=t_{f}+\Delta t$.

Let us consider the augmented system (6) with the following state feedback tracking controller:

$$
u(t)=K \bar{x}(t)=\left[K_{e}, K_{x}\right]\left[\begin{array}{l}
\varepsilon(t) \\
x(t)
\end{array}\right]
$$

The closed-loop augmented system is given by:

$$
\dot{\bar{x}}(t)=(\bar{A}+\bar{B} K) \bar{x}(t)+G y_{r}(t)
$$

The effective fault-tolerant controller which holds the tracking problem and minimizes the proposed control index (23) can be obtained by the following lemma.

Lemma 2: Consider the closed-loop augmented system (25) and the performance index (23). The following controller stabilizes the closed-loop augmented system for a given positive constant $\gamma$ :

$$
K=\left[K_{e}, K_{x}\right]=X Z^{-1}
$$

such the linear matrix inequality:

$$
\left[\begin{array}{cccc}
\bar{A} Z+\bar{B}_{f} X & G & X^{T} \omega \overline{\mathcal{R}}^{1 / 2} & Z Q^{1 / 2} \\
+\left(\bar{A} Z+\bar{B}_{f} X\right)^{T} & & 0 & 0 \\
* & -\gamma I & 0 & 0 \\
* & * & -I & -I
\end{array}\right] \prec 0
$$

where $Z \in \mathbb{R}^{(n+l) \times(n+l)}, \quad X \in \mathbb{R}^{m \times(n+l)}, Q=$ $\operatorname{diag}\left[Q_{1}, Q_{2}\right] \geq 0$ and $\overline{\mathcal{R}} \succ 0$.

Moreover, the upper bound of performance index $\mathcal{J}_{t}^{*}$ can be minimized by solving the following optimization problem

$$
\min \operatorname{Trace}(T) \text { s.t. }(27) \text { and }\left[\begin{array}{cc}
T & I \\
I & Z
\end{array}\right]
$$

where $T \in \mathbb{R}^{(n+l) \times(n+l)}$.

In the nominal case, the problem is solved for $\omega=I_{m}$ and $B_{f}=B$. After fault occurrence, the controller $K$ is calculated for the considering faulty actuator $\omega_{i} \neq 1$.

Proof: For the nominal case, the proof can be found in [13] by considering $\omega=I_{m}$ and $B_{f}=B$ in the constraint (27). For the degraded functional mode and after faults occurrence, the result follows by considering the value of loss control effectiveness $\omega$.

Indeed, in nominal functional mode, the weighting matrix $\overline{\mathcal{R}}$ is proposed based on the baseline actuators failure rates $\lambda_{i}^{0}$. After faults occurrence, a new values of actuators failure rates can be estimated on-line taken into account the applied load as illustrated in (9). Then, a new value of the weighting matrix $\overline{\mathcal{R}}$ is obtained and the fault-tolerant controller is calculated subject to the constraint (27).

\section{FLIGHT CONTROL APPLICATION}

In this section, an example of tracking control for a linear F-16 aircraft model is given to demonstrate the proposed approach. The linearized aircraft model is described as in (1), where, $x(t)=\left[u_{x}, u_{z}, q, u_{y}, p, r_{a}\right]^{T}$ is the state, $u(t)=\left[\delta_{h r}, \delta_{h l}, \delta_{a r}, \delta_{a l}, \delta_{r}\right]^{T}$ is the control input, and $y(t)=\left[a, \dot{\mu}, r_{s}, \alpha, \bar{\beta}\right]^{T}$ is the measured output, respectively. $u_{x}, u_{y}$ and $u_{z}$ are components of aircraft velocity along $X_{a}, Y_{a}$ and $Z_{a}$ body axes respectively. $p, q$ and $r_{a}$ are the roll rate about $X_{a}$ body axis, the pitch rate about the $Y_{a}$ body axis, and the yaw rate about $Z_{a}$ body axis, respectively. $\delta_{h r}, \delta_{h l}, \delta_{a r}, \delta_{a l}, \delta_{r}$ are right horizontal stabilator, left 
TABLE I

FAILURE RATES OF ELEMENTARY COMPONENTS

\begin{tabular}{|c|c|}
\hline \multicolumn{2}{|c|}{ Failure rates } \\
\hline$\lambda_{1}^{0}$ & $0.9 \mathrm{~min}^{-1}$ \\
\hline$\lambda_{2}^{0}$ & $0.4 \mathrm{~min}^{-1}$ \\
\hline$\lambda_{3}^{0}$ & $0.1 \mathrm{~min}^{-1}$ \\
\hline$\lambda_{4}^{0}$ & $0.05 \mathrm{~min}^{-1}$ \\
\hline$\lambda_{5}^{0}$ & $0.01 \mathrm{~min}^{-1}$ \\
\hline
\end{tabular}
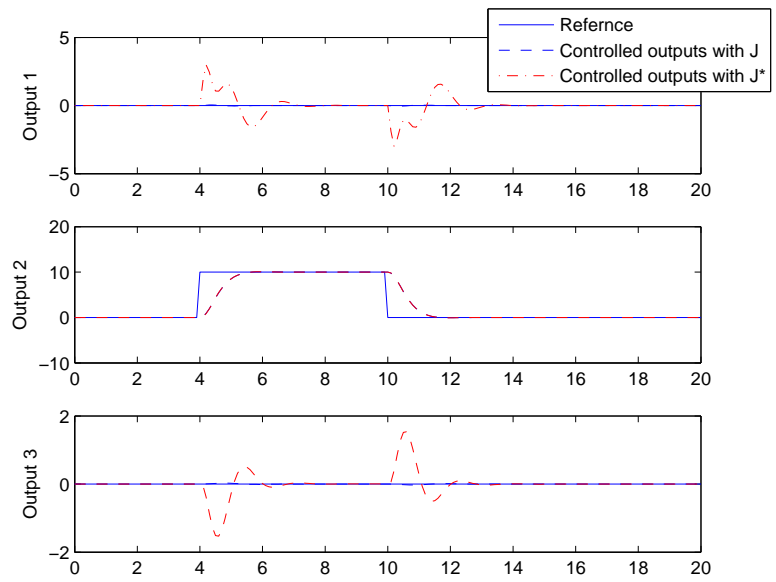

Fig. 1. Aircraft trajectory in the nominal case

horizontal stabilator, right aileron, left aileron, and rudder, respectively. $\dot{\mu}$ is the stability-axis roll rate and $r_{a}$ is the stability-axis yaw rate. $\alpha$ is angle of attack and $\bar{\beta}$ is angle of sideslip. The matrices, $A, B$ and $C$ are given in [7] (Example 1). For the degraded situations, each of the five actuators may lose its effectiveness. For the desired system dynamic and as chosen in [13], we consider $\gamma=2$ with:

$$
\mathrm{S}=\left[\begin{array}{lllll}
0 & 1 & 0 & 0 & 0 \\
0 & 0 & 0 & 1 & 0 \\
0 & 0 & 0 & 0 & 1
\end{array}\right]
$$

$Q_{1}=\operatorname{diag}[0.09,1,0.36], Q_{2}=\operatorname{diag}[0.01,0.2,0.01,0,0,0]$ where the matrix $S$ determines the output required to track, i.e., $\dot{\mu}, \alpha$ and $\beta$.

To illustrate the proposed approach in the short time window, the values of the actuators failure rates are adapted with the time of the considered scenario. The failure rates are considered with a very huge values and given in Table 1.

First, in the nominal case, two nominal controllers are calculated to guarantee the tracking requirement by solving the LMI problem (27). A nominal controller is obtained subject to the classical performance index (7) where the weighting matrix $\overline{\mathcal{R}}=\operatorname{diag}([0.49,0.49,0.004,0.04,0.04])$ is chosen as in [13]. Moreover, an effective nominal controller is calculated by considering the proposed performance index (23) where the matrix $\overline{\mathcal{R}}=\operatorname{diag}([0.10,0.54,0.35,0.60,1.30])$ is obtained based on the actuators failure rates $\lambda_{i}^{0}$. The parameter $\sigma=1.1$ is chosen in order to obtain a closedloop dynamic close to the classical one. In fact, the nominal
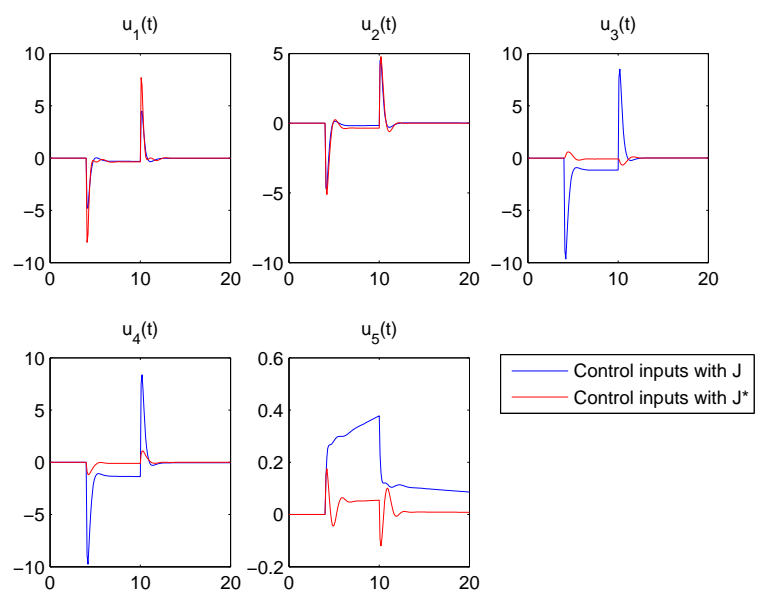

Fig. 2. Control inputs in the nominal case
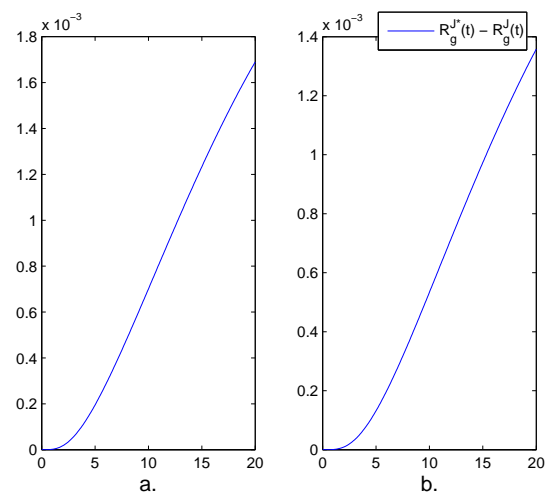

Fig. 3. Comparison between the reliabilities

controllers are calculated without loss of control effectiveness $\omega=I_{5}$. (The Yalmip solver is used in this application).

The effective nominal controller is designed in order to manage smartly the set of the actuators by considering the actuator health level. The actuator rate limits are not taken into account in the design. The Figure (1) shows the trajectory aircraft in the nominal case for the proposed performance index and for the classical one. It can be seen that the controlled outputs track the desired outputs without steadystate error. In Figure (2) the generated control inputs are presented where as can be seen clearly, the sensitive actuators are less solicited compared to the classical controller. In the same way, the required efforts are distributed to the less sensitive actuators in order to guarantee the stability and the tracking requirement. In fact, as presented in the Table 1, the set of the ordered actuators (14) is obtained from the less sensitive to the most sensitive one as: $l^{0}=$ $\left\{\lambda_{5}^{0}, \lambda_{4}^{0}, \lambda_{3}^{0}, \lambda_{2}^{0}, \lambda_{1}^{0}\right\}$. As consequence, the effective controller minimizes the load applied to the actuators 5,4 and 3 . The required efforts are compensated by the solicitation of the actuators 1 and 2. For the overall system reliability, it can be seen that the reliability of the system with the proposed controller $R^{*}(t)$ is more than in the classical one $R(t)$ where 

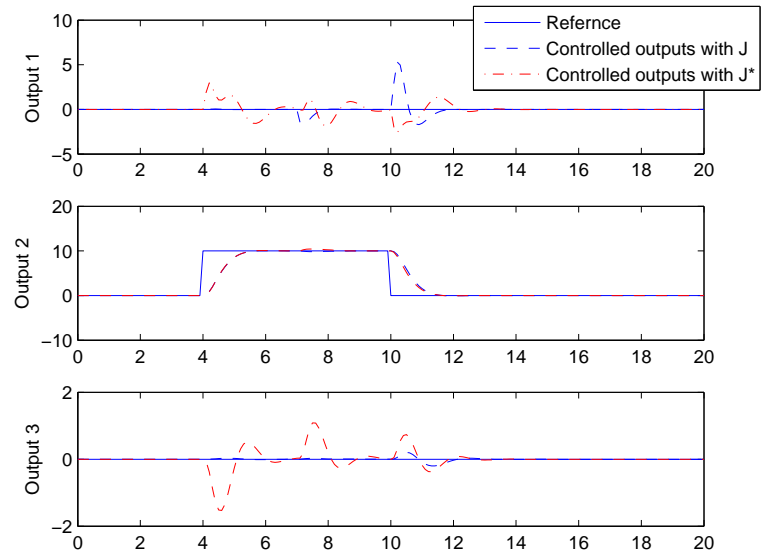

Fig. 4. Aircraft trajectory in the faulty case

Figure (3.a) shows the comparison between the reliabilities $R^{*}(t)-R(t)$. In fact, this result is evident where the sensitive actuators which cause an important reliability degradation of the overall system are less used.

For the faulty situation, we consider a failure of the actuator 3 at $t=7 \mathrm{sec}$. This degraded mode can be characterized by $\omega_{3}=0$ from the time of fault occurrence. The controller is calculated in order to find a novel distribution of the desired efforts that tracks the reference and stabilizes the system taken into consideration the impact of the applied load on the health level associated to each actuator. For the proposed index performance, the control efforts are distributed with respect to the actuators health where a new estimation of the failure rates $\lambda_{i}$ using (9) is considered in the performance index (23). The aim is to obtain a high overall system reliability by minimizing the use of the sensitive actuators taking into account the health degradation caused by the applied load before reconfiguration.

The Figure (4), shows the outputs responses in the faulty case. It can be seen that even the presence of fault, the controlled outputs track the desired outputs without steadystate error. In Figure (5), the control inputs are presented both for the classical controller compared to the proposed controller. Again, the use of the most sensitive actuators and the faulty one is minimized. This distribution maximizes the overall system reliability and improves the dependability of the reconfigurable system where, as shown the Figure(3.b), the life time of the proposed configuration is greater. This result means that the probability that the system remains operational is larger.

\section{CONCLUSION}

In this work, a fault-tolerant control design based on actuators health analysis is proposed. The aim is to contribute to a reliable reconfigurable systems where the distribution of the desired efforts is performed by informations about the actuators reliability in addition to the occurred faults. The obtained results show the performance of the proposed approach in term of overall system reliability and the life
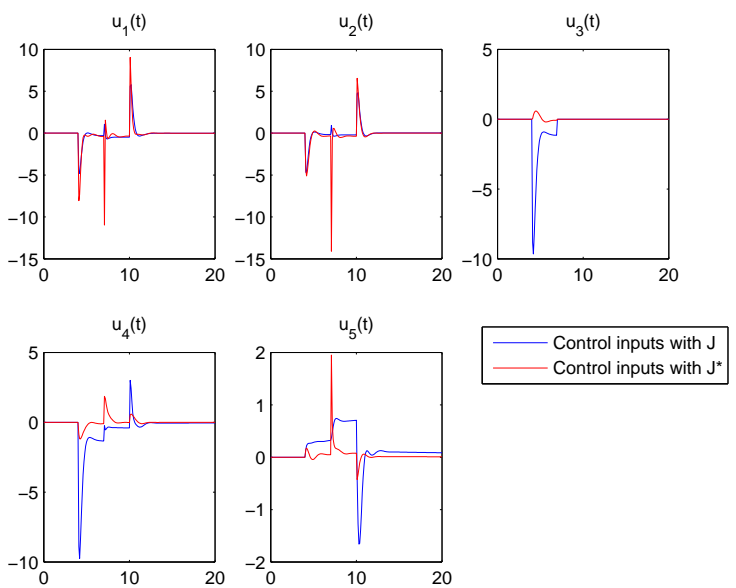

Fig. 5. Control inputs in the faulty case

time of the reconfigurable system. In fact, an effective management of the desired control inputs taking into account the actuators health improves the overall system reliability and the system dependability by keeping the system operational longer in time.

\section{REFERENCES}

[1] M. Blanke, M. Kinnaert, J. Lunze, and M. Staroswiecki. Diagnosis and Fault Tolerant Control. Springer-Verlag London, 2006.

[2] D.R. Cox. Regression models and life tables. Journal of the Royal Statistical Society, vol. 34, pp 187-220, 1972.

[3] I. Gertsbakh. Reliability Theory with Applications to Preventive Maintenance. Springer, 2000

[4] F. Guenab and P. Weber and D. Theilliol and Y. Zhang. Design of a fault tolerant control system incorporating reliability analysis under dynamic behaviour constraints. In International Journal of Systems Science, vol. 42, no. 1, pp 219-223, 2010.

[5] X. He and Z. Wang and D. Zhou. Robust $H_{\infty}$ filtering for timedelay systems with probabilistic sensor faults. IEEE Signal Processing Letters, vol. 16, pp 442-445, 2009.

[6] A. Khelassi and J. Jiang and D. Theilliol and P. Weber and Y.M. Zhang. Reconfiguration of control inputs for over-actuated systems based on actuators health. In Proceedings of 18th IFAC World Congress, Milan, Italy, 2011.

[7] F. Liao and J. L. Wang and G. Y. Reliable robust flight tracking control: An LMI approach. IEEE Transactions on Control Systems Technology, vol. 10, no. 1, 2002.

[8] H. Noura and D. Theilliol and J.C. Ponsart, and A. Chamssedine. Fault Tolerant Control Systems: Design and Practical Application. Springer Dordrecht Heidelberg London, 2009.

[9] M. Staroswiecki and G. Hoblos. Sensor network design for fault tolerant estimation. International Journal of Adaptive Control Signal Precessing, vol. 18, pp 55-72, 2004.

[10] M. Staroswiecki and D. Berdjag. General fault tolerant control design. In. International Journal of Systems Science, vol. 41, pp 971-985, 2010.

[11] N.E. Wu. Reliability and fault tolerant control system Part II. IEEE Conference on Decision and control, pp 1466-1471, 2001, Orlando, Florida, USA

[12] N.E. Wu and R. J. Patton. Reliability and supervisory control. In $5^{\text {th }}$ IFAC Symposium on fault Detection, Supervision ans Safety of Technical Processes,pp 139-144, 2003, Washington DC, USA.

[13] D. Ye and G. Yang. Adaptive fault tolerant tracking control against actuator faults with application to flight control. IEEE Transactions on Control Systems Technology, vol. 14, pp 1088-1096, 2006.

[14] Y.M. Zhang and J. Jiang. Bibliographical review on reconfigurable tolerant-control system. Annual Reviews in Control, vol. 32, pp 229252, 2008. 